\title{
Distribución espacial de epifitos en el talo de la macroalga introducida Codium fragile subsp. tomentosoides en el submareal de Caldera
}

Spatial distribution of epiphytes along the thallus of the introduced macroalga, Codium fragile subsp. tomentosoides in the subtidal zone of Caldera

\section{Cristóbal Villaseñor-Parada ${ }^{1}$ y Paula E. Neill $^{1}$}

\begin{abstract}
${ }^{1}$ Departamento de Ecología, Facultad de Ciencias, Universidad Católica de la Santísima Concepción, Casilla 297, Concepción, Chile.pneill@ucsc.cl
\end{abstract}

\begin{abstract}
Codium fragile subsp. tomentosoides is an introduced macroalga on the coast of Chile. We identified and quantified the epiphytes present in basal, intermediate and apical sections of 32 thalli. The accumulation of epiphytes over time would explain why older sections of the thallus (basal and intermediate sections) host a greater amount of epiphytes, and why the composition of epiphyte assemblages in these sections is similar. In contrast, newer sections (apical) have been available for colonization for less time, resulting in a lower epiphyte load, and the composition of assemblages from this section differs from that of the rest of the thallus.
\end{abstract}

Key words: Epiphyte load, colonization, introduced species, biological substrate

\section{INTRODUCCIÓN}

Muchos organismos marinos, tanto en ambientes intermareales como submareales, utilizan sustratos biológicos para su asentamiento (e.g., invertebrados, macroalgas, pastos marinos). La distribución de los epibiontes en sus organismos hospedadores no siempre es al azar, sino que más bien obedece a patrones definidos, que pueden estar relacionados con los requerimientos de los propios epibiontes (e.g., cantidad de luz, exposición a corrientes, flujo de agua; Borowitzca et al. 1990, Romagnoli et al. 2007), con respuestas evolutivas a interacciones bióticas (e.g., herbivoría, Durante \& Chia 1991), o bien, con la acumulación de epibiontes a través del tiempo (Borowitzca et al. 1990, Trautman \& Borowitzca 1999).

Codium fragile (Suringar) Hariot subsp. tomentosoides (van Goor) Silva, es una macroalga verde, reconocida como una especie invasora en ecosistemas marinos en varias partes del mundo (Trowbridge 1998, 1999, Mathieson et al. 2003, Nyberg \& Wallentinus 2005). Esta especie posee una particular morfología de talo tridimensional formado por un sistema de filamentos sifonosos que generan espacios e intersticios en el talo, que favorecen el asentamiento de algas e invertebrados (Trowbridge 1998, 1999), registrándose una gran cantidad de epifitos [i.e., cualquier organismo que habite sobre un organismo fotosintético, existiendo o no una relación nutricional entre ambos (Harlin 1980, Steel \& Wilson 2003)].
Los estudios realizados en epifitos de Codium fragile se han focalizado principalmente en las consecuencias ecológicas que éstos tienen sobre su hospedador, tales como herbivoría (Trowbridge 1993, 1998, 1999), crecimiento y probabilidad de desprendimiento por fuerza del oleaje (Bégin \& Scheibling 2003), pero los patrones de distribución espacial de estos epifitos, así como las causas que originan dichos patrones, no han sido evaluados.

En Chile, Codium fragile se extiende desde el norte chico hasta el extremo austral, con una distribución discontinua y parchosa (Neill et al. 2006). Si bien, en Tierra del Fuego existen reportes de su existencia desde 1847, en el norte de Chile, su aparición es más reciente, reportándose como plaga en 1998 por trabajadores de acuicultura (Neill et al. 2006). En Caldera, Región de Atacama, esta macroalga coloniza zonas intermareales y submareales someras, albergando una alta carga epifita (González \& Santelices 2004). El objetivo del presente trabajo fue evaluar si existe un patrón en la distribución de epifitos en el talo de $C$. fragile en la costa de Caldera, donde $C$. fragile ha sido introducida, lo que podría servir como una primera aproximación para comprender los tipos de interacciones que esta macroalga tiene con su comunidad receptora. 


\section{Materiales y MÉTODOS}

Se recolectaron 32 ejemplares de Codium fragile subsp. tomentosoides, de una longitud total de 30,4 $\pm 10,35 \mathrm{~cm}$ (promedio \pm desviación estándar). Dicha recolección se realizó en la zona submareal somera al noreste de Bahía

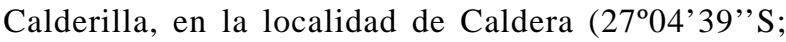
$70^{\circ} 50$ '70”O), entre julio del 2004 y agosto del 2005 (dos o tres talos por mes), los cuales se mantuvieron congelados hasta el momento de ser analizados.

Los talos de Codium fragile fueron divididos en tres secciones: basal, media y apical. En cada sección se obtuvieron tres fragmentos del talo, de cinco centímetros cada uno (Fig. 1), los que fueron revisados bajo lupa óptica para la determinación taxonómica de las algas e invertebrados sésiles epifitos, llegando hasta el nivel taxonómico más bajo posible. Adicionalmente, se estimó la abundancia de cada epifito como el porcentaje de cobertura ocupado en el fragmento, empleando para ello una escala ordinal con valores que van desde 0 (ausencia del epifito) a 20 (total cobertura del epifito). A partir de estos valores se calculó el índice de diversidad de Shannon-Wiener (H') para cada fragmento. En las observaciones, no se consideraron los invertebrados móviles, ni microorganismos.

Para comparar riqueza, diversidad y cobertura de epifitos entre las tres secciones del talo, se utilizó un diseño experimental en bloques, agrupando las unidades experimentales (fragmentos) en bloques (talos), para así poder comparar los tratamientos (sección del talo) en un medio más homogéneo y evitar posibles sesgos producidos por características propias de cada talo (e.g., tamaño, edad) (Zar 1996). En los casos de riqueza y cobertura, los datos fueron transformados a raíz cuadrada para poder cumplir con los supuestos de normalidad y homocedasticidad (Zar 1996). Se utilizó la prueba de Kolmogorov-Smirnov para verificar el supuesto de normalidad, y la prueba de Cochran para evaluar homocedasticidad. Se aplicó un ANDEVA en bloque y luego una prueba a posteriori de Tukey.

Para evaluar diferencias en la composición comunitaria de epifitos en las tres secciones del talo, se construyó una matriz de similitud basada en la medida de distancia de Bray-Curtis, con los datos transformados a $\log _{(\mathrm{n}+1)}$, a la cual se aplicó una prueba de ANOSIM anidado, la cual permite detectar diferencias en composición comunitaria entre grupos (secciones), dentro de cada talo, mediante permutaciones realizadas a la matriz original (Clarke 1993). Además, se aplicó el análisis de Similitud Porcentual (SIMPER), para determinar cuáles taxa fueron los principales responsables de las diferencias encontradas (Clarke 1993).

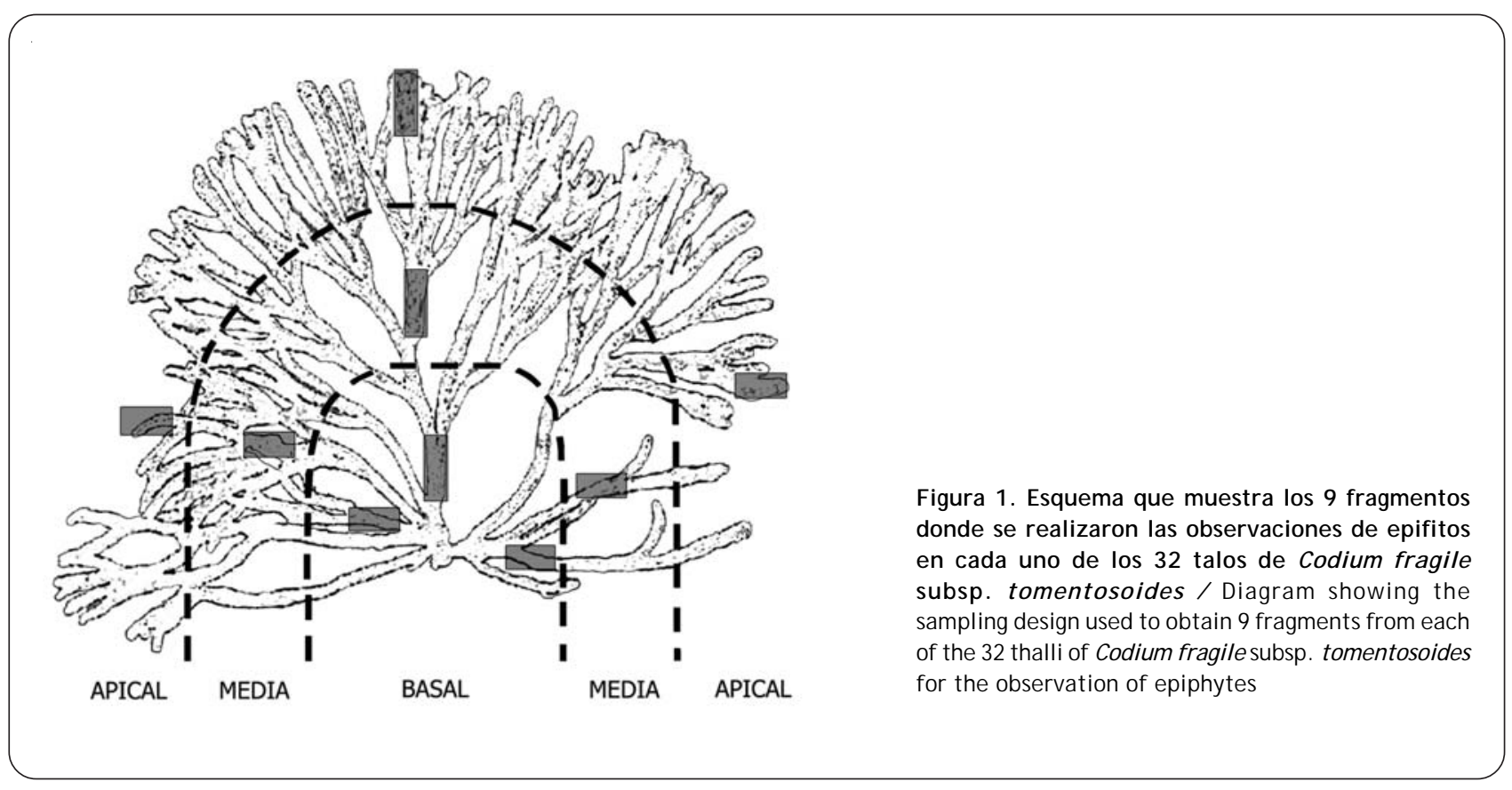




\section{RESULTADOS Y DISCUSIÓN}

Un total de 9 algas y 6 tipos de invertebrados sésiles fueron identificados en los 32 talos analizados de Codium fragile subsp. tomentosoides. La mayoría de las algas identificadas pertenecían a la división Rhodophyta, y en menor frecuencia ejemplares de las divisiones Chlorophyta y Cyanobacteria, patrón que también se ha observado a otras latitudes (e.g., Mathieson et al. 2003, Schmidt \& Scheibling 2006, Jones \& Thornber 2010). En el caso de algunos invertebrados sésiles, no se pudo llegar a nivel de género o especie, por lo que fueron determinados a niveles taxonómicos mayores.

De acuerdo a la frecuencia de ocurrencia en los talos analizados, se reconocieron cuatro tipos de epifitos, clasificados en: i) muy frecuentes (encontrados en más de 20 talos), ii) frecuentes (entre 10 y 19 talos), iii) poco frecuentes (entre 2 y 9 talos) y iv) ocasionales (solamente en 1 talo) (Tabla 1). Las algas filamentosas Neosiphonia harveyi (Bailey) Kim, Choi, Guiry \& Saunders y Acrochaetium sp. fueron los epifitos más conspicuos. Siete especies de epifitos fueron encontradas en sólo un talo durante todo el período de estudio, a pesar de que algunas de ellas (e.g., foraminíferos, espirórbidos, mitílidos, Cladophora sp.) son muy comunes en la comunidad submareal de Bahía Calderilla (Neill 2007). Estas especies fueron definidas como 'ocasionales' en el talo de Codium fragile, por lo tanto no fueron consideradas en los análisis.

En cuanto a la distribución espacial de los epifitos en el talo de Codium fragile, a excepción de las especies ocasionales, todas fueron encontradas en las tres secciones del talo, lo que demuestra su capacidad para asentarse en cualquier sector de la macroalga. Sin embargo, en la mayoría de los casos la frecuencia de ocurrencia fue menor en la sección apical (Tabla 1). Además, el ANDEVA en bloque mostró diferencias significativas en riqueza y diversidad de epifitos entre las tres secciones del talo, con altos valores en la sección basal, disminuyendo gradualmente hacia la sección apical (Figs. 2a y 2c). Considerando que $C$. fragile presenta un crecimiento localizado en el ápice (Trowbridge 1999), existe una relación directa entre cantidad de epifitos (i.e., riqueza, diversidad y cobertura), y la edad del sector del

Tabla 1. Especies de epifitos en 32 talos de C. fragile, clasificados según su frecuencia de ocurrencia, la cantidad de talos en los cuales fueron observados (entre paréntesis) y el número de veces en que el epifito fue observado en cada una de las tres secciones analizadas / Epiphyte species found on the $32 \mathrm{C}$. fragile thalli, classified according to their frequency of occurrence, the number of thalli in which a particular species was observed (in parentheses), and the number of times a species was observed in each of the 3 analyzed sections

\begin{tabular}{lccccc}
\hline \multirow{2}{*}{ Especies } & División/ & Frecuencia de ocurrencia & \multicolumn{3}{c}{ Distribución espacial } \\
& Phylum & & Apical & Media & Basal \\
\hline Neosiphonia harveyi & Rhodophyta & Muy Frecuente (30) & 29 & 28 & 28 \\
Acrochaetium sp. & Rhodophyta & Muy Frecuente (28) & 10 & 26 & 21 \\
Ulva spp. & Chlorophyta & Frecuente (15) & 5 & 10 & 11 \\
Bryozoa sp.1 & Bryozoa & Frecuente (15) & 4 & 8 & 13 \\
Hydrozoa sp.1 & Cnidaria & Frecuente (10) & 1 & 7 & 8 \\
Chondria sp. & Rhodophyta & Poco Frecuente (7) & 2 & 1 & 5 \\
Cyanobacteria sp.1 & Cyanobacteria & Poco Frecuente (6) & 3 & 1 & 4 \\
Acrosorium sp. & Rhodophyta & Poco Frecuente (6) & 1 & 3 & 4 \\
Balanus laevis & Arthropoda & Poco Frecuente (6) & 0 & 0 & 6 \\
Foraminifera sp.1 & Foraminiferida & Ocasional (1) & 0 & 1 & 1 \\
Mytilidae sp.1 & Mollusca & Ocasional (1) & 1 & 0 & 0 \\
Espirorbidos & Annelida & Ocasional (1) & 0 & 0 & 1 \\
Rhodymenia sp. & Rhodophyta & Ocasional (1) & 0 & 0 & 1 \\
Ceramium sp. & Rhodophyta & Ocasional (1) & 0 & 0 & 1 \\
Cladophora sp. & Chlorophyta & Ocasional (1) & 0 & 0 & 1 \\
\hline
\end{tabular}



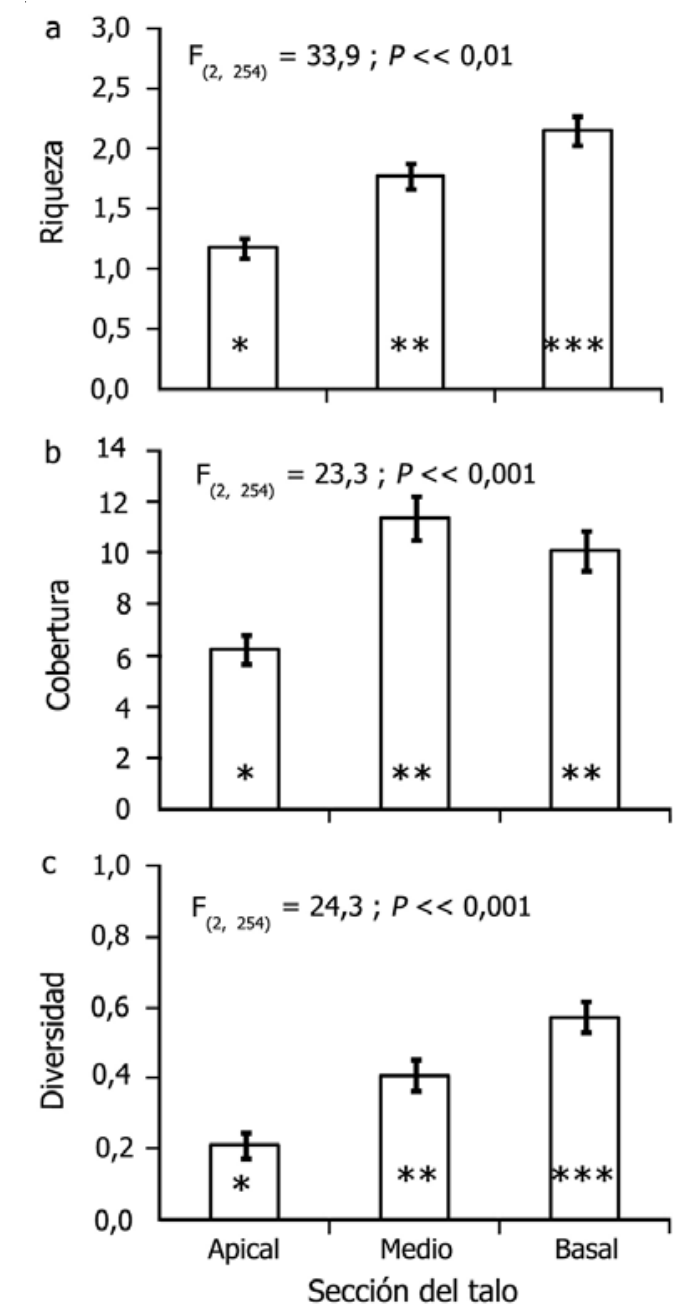

Figura 2. Riqueza (a), cobertura (b) y diversidad (c) de especies epifitas en las tres secciones del talo de $C$. fragile (promedio \pm error estándar). Los resultados del ANDEVA en bloque se muestran para cada caso. Asteriscos $(*, * *, * * *)$ en las columnas identifican grupos heterogéneos de acuerdo a la prueba a posteriori de Tukey / Richness (a), cover (b) and diversity (c) of epiphytic species on the three sections of $\mathrm{C}$. fragile thalli (average \pm standard error). The results of the randomized block ANOVA are shown for each case. Asterisks (*,**,***) on the columns indicate heterogeneous groups based on Tukey's post hoc test

talo, es decir, los sectores más nuevos de la macroalga albergan una menor cantidad de epifitos, respecto a los sectores más viejos. En cuanto a la cobertura de epifitos, esta resultó ser estadísticamente diferente sólo en la sección apical, pero similar entre las secciones basal y media (Fig. 2b), evidenciando que el sector más joven de la macroalga presenta una mayor superficie disponible para ser colonizado. Los bloques (talos) en los análisis de ANDEVA fueron significativos tanto para riqueza $\left(\mathrm{F}_{(31}\right.$, $\left.{ }_{254)}=39,9 ; P<0,001\right)$, diversidad $\left(\mathrm{F}_{(31,254)}=23,3 ; P<0,001\right)$ y cobertura $\left(\mathrm{F}_{(31,254)}=24,3 ; P<0,001\right)$.

En relación a la composición comunitaria de los ensambles de epifitos, la prueba global de ANOSIM, detectó diferencias significativas entre las secciones del talo $\left(\mathrm{R}_{\text {ANosim }}=0,07 ; P=0,001\right)$, donde pruebas pareadas mostraron que los ensambles de la sección apical son diferentes a los ensambles de las secciones basal ( $\mathrm{R}_{\text {ANosim }}$ $=0,11 ; P=0,001)$ y media $\left(\mathrm{R}_{\text {Anosim }}=0,09 ; P=0,001\right)$, mientras que los ensambles de las secciones media y basal son estadísticamente similares entre sí $\left(\mathrm{R}_{\text {ANosim }}=\right.$ 0,009; $P=0,78)$. La mayor cobertura de Neosiphonia harveyi en la sección apical, y la mayor cobertura de Acrochaetium sp. en las secciones media y basal, son las principales responsables de las diferencias encontradas en la composición de ensambles (SIMPER).

Los resultados de este estudio apoyan la idea de que la distribución de epifitos en el talo de Codium fragile subsp. tomentosoides no es al azar, sino que más bien obedece a patrones definidos, particularmente a la acumulación temporal de epifitos. Además, observaciones en terreno sugieren que la colonización de epifitos es constante a través del tiempo (i.e., los epifitos se encuentran en talos de todos los tamaños, con alta carga epifita durante todo el año, y además, la presión de propágulos de epifitos es constante durante el año). Basado en los resultados obtenidos, se propone que, en el submareal somero de Bahía Calderilla, los propágulos de algas e invertebrados sésiles llegan constantemente al talo de C. fragile, siendo los sectores del talo más viejos (sección basal) los que albergan mayor cantidad de epifitos (mayor riqueza, diversidad y abundancia). Ensambles de epifitos de las secciones basal y media son similares, ya que llevan mayor tiempo siendo colonizados, la sección apical en cambio es más nueva, por lo que tiene menor cobertura de epifitos, es decir mayor superficie disponible para el asentamiento, la que iría aumentando con el tiempo, haciéndose más similar a las otras dos secciones.

$\mathrm{Al}$ igual que en otras localidades donde Codium fragile subsp. tomentosoides ha sido introducida (e.g., Trowbridge 1998, 1999, Mathieson et al. 2003, Bégin \& Scheibling 2003, González \& Santelices 2004, Schmidt \& Scheibling 2006, Jones \& Thornber 2010), en Caldera, el alga roja Neosiphonia harveyi (Polysiphonia harveyi) es el epifito más conspicuo en el talo de $C$. fragile. Esta macroalga fue reportada para Chile por primera vez por 
Leonardi et al. (2006), como epifito sobre Gracilaria chilensis en cultivos del norte de Chile, y a pesar de no encontrarse en los listados de especies exóticas de Castilla et al. (2005) y Castilla \& Neill (2009), se plantea como una probable especie introducida en Chile, basado en su origen asiático, su amplia distribución mundial y su asociación con vectores antrópicos (Mathieson et al. 2008). No se descarta una posible interacción positiva entre $N$. harveyi y $C$. fragile que permitiría la introducción de la primera, a raíz de la introducción de la segunda (e.g., facilitación sensu Bruno et al. 2005; 'invasional meltdown' sensu Simberloff \& Von Holle 1999), tal como se ha observado en la asociación Codium fragileMembraniphora membranacea (Levin et al. 2002).

Debido a que Codium fragile ha sido considerada como una 'peligrosa' especie invasora a nivel mundial, y es poco conocida y estudiada en las costas chilenas, resulta de especial interés conocer y predecir sus potenciales efectos en su comunidad. En este trabajo se observó que $C$. fragile, debido a su morfología, es un potencial sustrato secundario para gran cantidad de organismos. En estudios anteriores, se ha determinado que la presencia de $C$. fragile, como especie exótica, afecta negativamente a algunas de las especies que se asientan en la superficie rocosa, como Acrochaetium sp., Ulva spp. y Briozoa sp.1 (Neill 2007), la presencia de esta alga no necesariamente lleva a la extinción local de especies nativas, sino que más bien, las excluye o disminuye sus abundancias relativas en sustratos primarios (Bruno et al. 2005). En este sentido, el entender los mecanismos que dan origen a la distribución espacial de epibiontes en sustratos biológicos puede ser de utilidad como una primera aproximación para identificar con mayor precisión, el verdadero impacto de especies exóticas, sobre la biodiversidad, tanto en interacciones entre especies y facilitación en la introducción de nuevas especies, sobre todo en los casos donde la especie exótica constituye un sustrato novedoso en la comunidad local.

\section{Agradecimientos}

Los autores agradecen a Jessica Beltrán, Verónica Flores, y Macarena Schmidt por apoyo con la identificación de epifitos, a Sylvain Faugeron y Oscar Alcalde por su apoyo en terreno, a Sergio Navarrete y Juan Correa por el apoyo prestado durante la realización de la tesis doctoral de Paula Neill, y a tres revisores anónimos por sus valiosos comentarios y sugerencias. La recolección de muestras fue financiada por CONICYT a través de una Beca de Apoyo para la Realización de Tesis Doctoral N²4040050 y una beca de P.E.O. chapter C-Z a Paula Neill, mientras que el análisis de los datos fue financiado parcialmente a través del proyecto FONDECYT Nº 11070183.

\section{LITERATURA CITADA}

Bégin C \& RE Scheibling. 2003. Growth and survival of the invasive green alga Codium fragile ssp. tomentosoides in tide pools on a rocky shore in Nova Scotia. Botanica Marina 46: 404-412.

Borowitzca MA, RC Lethbridge \& L Charlton. 1990. Species richness, spatial distribution, and colonisation pattern of algal and invertebrate epiphytes on the seagrass Amphibolis griffithii. Marine Ecology Progress Series 64: 281-291.

Bruno JF, JD Fridley, K Bromberg \& MD Bertness. 2005. Insights into biotic interactions from studies of species invasions. En: Sax DF, JJ Stachowicz \& SD Gaines (eds). Species invasions: Insights into ecology, evolution, and biogeography, pp. 13-40 Sinauer, Sunderland.

Castilla JC \& PE Neill. 2009. Marine bioinvasions in the southeastern Pacific: status, ecology, economic impacts, conservation and management. En: Rilov G \& JA Crooks (eds). Biological invasions in marine ecosystems, pp. 439457. Springer, Berlin.

Castilla JC, M Uribe, $\mathbf{N}$ Bahamonde, $M$ Clarke, $\mathbf{R}$ Desqueyroux-Faúndez, I Kong, H Moyano, N Rozbaczylo, B Santelices, C Valdovinos \& P Zavala. 2005. Down under the southeastern Pacific: marine non-indigenous species in Chile. Biological Invasions 7: 213-232.

Clarke KR. 1993. Non-parametric multivariate analyses of changes in community structure. Australian Journal of Ecology 18: 117-143.

Durante KM \& FS Chia. 1991. Epiphytism on Agarum fimbriatum: can herbivore preferences explain distributions of epiphytic bryozoans? Marine Ecology Progress Series 77: 279-287.

González A \& B Santelices. 2004. A dichotomous species of Codium (Bryopsidales, Chlorophyta) is invading northern Chile. Revista Chilena de Historia Natural 77: 293-304.

Harlin MM. 1980. Seagrass epiphytes. En: Phillips RC \& CP McRoy (eds). Handbook of seagrass biology, pp. 117-151. Garland STPM Press, New York.

Jones E \& CS Thornber. 2010. Effects of habitat-modifying invasive macroalgae on epiphytic algal communities. Marine Ecology Progress Series 400: 87-100.

Leonardi PI, AB Miravalles, S Faugeron, V Flores, J Beltrán \& JA Correa. 2006. Diversity, phenomenology and epidemiology of epiphytism in farmed Gracilaria chilensis (Rhodophyta) in northern Chile. European Journal of Phycology 41: 247-257.

Levin PS, JA Coyer, R Petrik \& TP Good. 2002. Communitywide effects of non-indigenous species on temperate rocky reefs. Ecology 83: 3182-3193. 
Mathieson AC, CJ Dawes, LG Harris \& EJ Hehre. 2003. Expansion of the Asiatic green alga Codium fragile subsp. tomentosoides in the Gulf of Maine. Rhodora 105(921): 1-53.

Mathieson AC, JR Pederson, CD Neefus, CJ Dawes \& TL Bray. 2008. Multiple assessments of introduced seaweeds in the Northwest Atlantic. ICES Journal of Marine Science 65: 730-741.

Neill PE. 2007. Distribución de la macroalga introducida Codium fragile (Chlorophyta) en Chile y sus efectos sobre la estructura de los ensambles del submareal rocoso del norte chico. Tesis Doctoral, Facultad de Ciencias Biológicas, Pontificia Universidad Católica de Chile, Santiago, 179 pp.

Neill PE, O Alcalde, S Faugeron, SA Navarrete \& JA Correa. 2006. Invasion of Codium fragile ssp. tomentosoides in northern Chile: a new threat for Gracilaria farming. Aquaculture 259: 202-210.

Nyberg CD \& I Wallentinus. 2005. Can species traits be used to predict marine macroalgal introductions? Biological Invasions 7: 265-279.

Romagnoli T, G Bavestrello, EM Cucchiari, M Stefano, CG Camillo, C Pennesi, S Puce \& C Totti. 2007. Microalgal communities epibiontic on the marine hydroid Eudendrium racemosum in the Ligurian Sea during an annual cycle. Marine Biology 151: 537-552.

Schmidt AL \& RE Scheibling. 2006. A comparison of epifauna and epiphytes on native kelps (Laminaria species) and an invasive alga (Codium fragile susp. tomentosoides) in Nova Scotia, Canada. Botanica Marina 49: 315-330.
Simberloff D \& B Van Holle. 1999. Positive interactions of nonidigenous species: invasional meltdown? Biological Invasions 1: 21-32.

Steel JB \& JB Wilson. 2003. Which is the phyte in epiphyte? Folia Geobotánca 38:97-99.

Trautman DA \& MA Borowitzca. 1999. Distribution of the epiphytic organisms on Posidonia australis and P. sinuosa, two seagrasses with differing leaf morphology. Marine Ecology Progress Series 179: 215-229.

Trowbridge CD. 1993. Interactions between an ascoglossan sea slug and its green algal host: branch loss and role of epiphytes. Marine Ecology Progress Series 101: 263-272.

Trowbridge CD. 1998. Ecology of the green macroalga Codium fragile (Suringar) Hriot 1889: invasive and non-invasive subspecies. Oceanography and Marine Biology: an Annual Review 36: 1-64.

Trowbridge CD. 1999. An assessment of the potential spread and options for control of the introduced green macroalga Codium fragile ssp. tomentosoides on Australia shores, 44 pp. Consultancy Report, Center for Research on Introduced Marine Pests, CSIRO, Hobart.

Zar JH. 1996. Biostatistical analysis, 867 pp. Prentice Hall, Englewood Cliffs.

Recibido el 15 de diciembre de 2010 y aceptado el 16 de marzo de 2011 